\title{
Bananen (Musa-Arten) - Riesenstauden mit Zier- und Nutzwert
}

\author{
VeIT M. DÖRKEN
}

\begin{abstract}
Banana plants are giant, tropical perennial herbs. Some species are cultivated for their fruits, others as ornamental solitary plants in gardens and parks. Musa basjoo is hardy enough for outdoor cultivation in Central Europe, where it also produces huge inflorescences and fruits.
\end{abstract}

\section{Zusammenfassung}

Bananenpflanzen sind tropische Riesenstauden, die wichtige Obstlieferanten und attraktive Solitärpflanzen darstellen. Die winterharte Musa basjoo eignet sich auch in Mitteleuropa zur Freilandkultur, wo sie im Sommer gigantische Blütenstände und auch Früchte hervorbringen kann.

\section{Einleitung}

Die Gattung Musa (Banane, Musaceae) umfasst knapp 40 Arten und wurde vom berühmten schwedischen Botaniker CARL vON LINNÉ (1707-1778) nach Antonius Musa, einem römischen Arzt (63-14 v. Chr.), benannt. Bananen stammen aus tropischen Regionen Asiens, wo sie ein wichtiges Element der Großstauden-

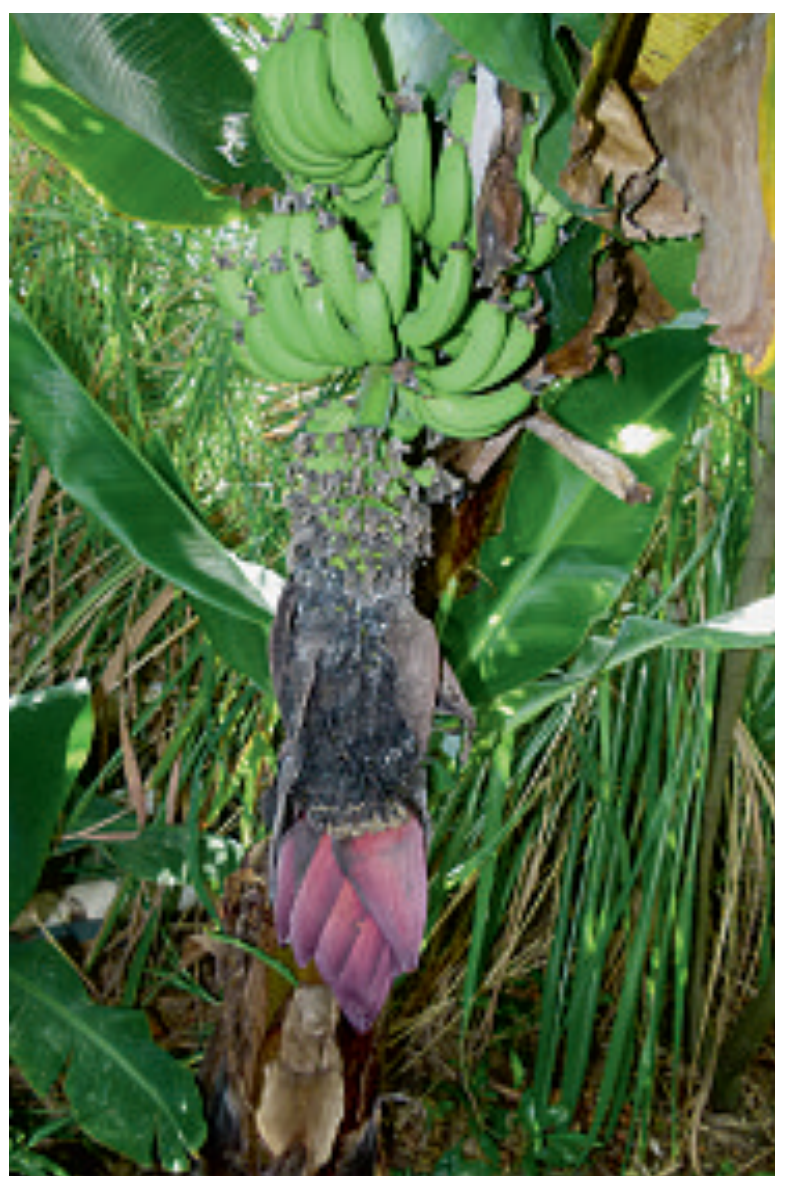

fluren in Wäldern sind. Am bekanntesten ist die Kulturbanane (Musa x paradisiaca) mit ihren wohlschmeckenden Früchten, wobei es auch Zierbananen mit schönen Blütenständen (und ungenießbaren Früchten) gibt.

Bananenstauden gehören zu den ältesten Kulturpflanzen überhaupt. Essbare Bananen stammen von den beiden Wildarten Musa acuminata und M. balbisiana ab. Vermutlich sind sie im feucht-tropischen Südost-Asien entstanden. Durch den Menschen wurden dort Pflanzen mit samenlosen Früchten ausgelesen, die ein essbares und weiches Fruchtfleisch haben. Von Asien gelangten Bananenpllanzen schon in vorchristlicher Zeit durch arabische Kaufleute nach Afrika. Portugiesen führten sie auf den Kanarischen Inseln ein und brachten sie in die Karibik. Durch die Spanier kamen sie im 16. Jh. nach Südamerika. Die Bezeichnung „Banan“, aus der später unsere „Banane“ wurde, stammt von den Arabern und bedeutet Finger, was sich auf die fingerförmig angeordneten Früchte bezieht.

\section{Morphologie}

\subsection{Vegetativer Bau}

Alle Bananen-Arten sind trotz ihres mitunter baumähnlichen, bis $10 \mathrm{~m}$ hohen Wuchses, krautige Pflanzen. Es handelt sich um Stauden mit einem unterirdischen, knollenförmigen Wurzelstock (Rhizom), mit dem die Pflanzen zur Ausläuferbildung und somit zur vegetativen Vermehrung befähigt sind. Das Rhizom kann 


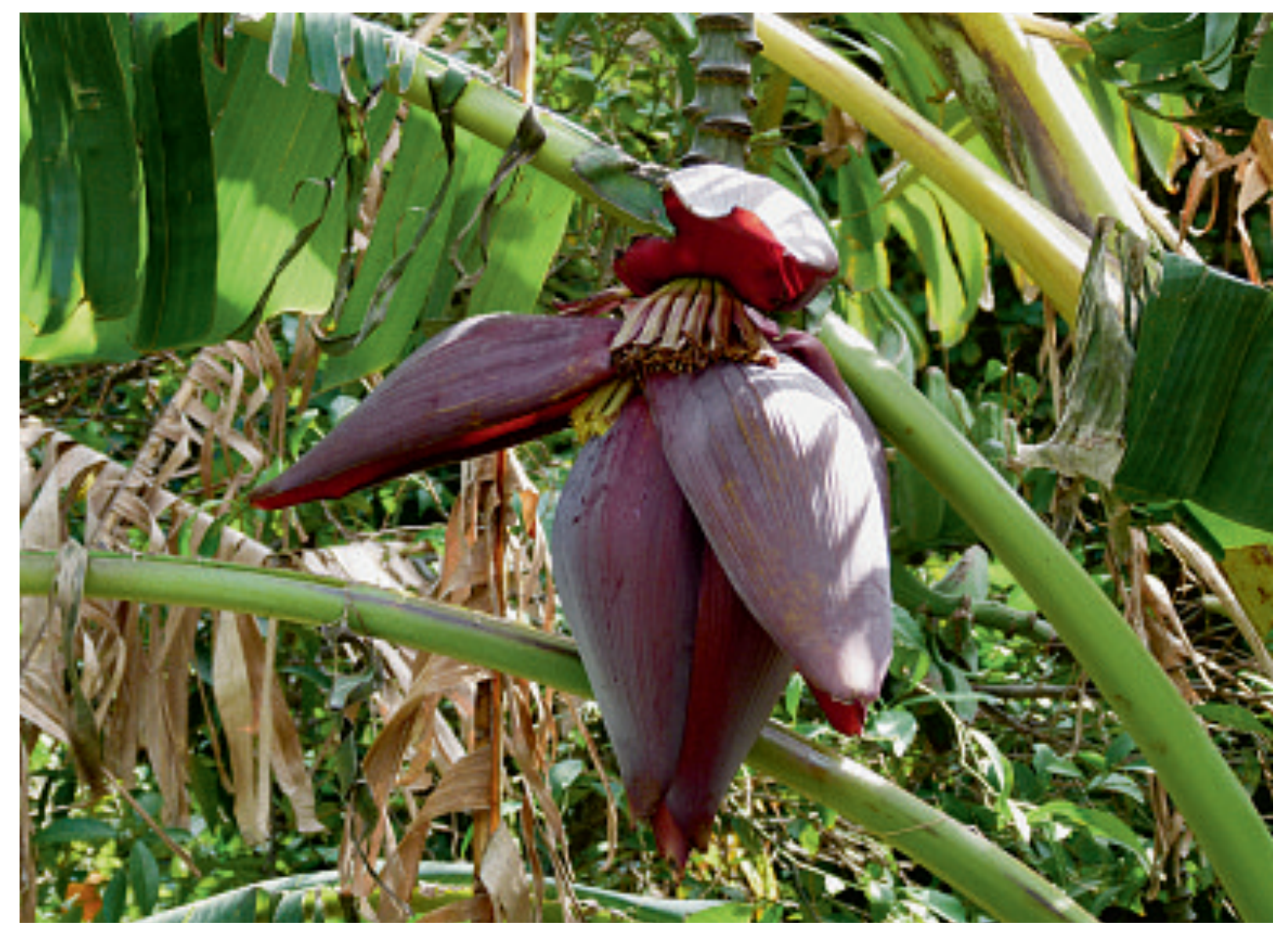

reichlich Nährstoffe speichern. Aus dem Wurzelstock entspringen die gewaltigen Blätter, deren Blatt-Scheiden eng umeinander gewickelt einen unverholzten Scheinstamm bilden. Der Querschnitt durch einen Scheinstamm erinnert entfernt an den Querschnitt durch eine Küchenzwiebel.

Während die jungen Blätter durch den Scheinstamm emporwachsen, sind sie eng eingerollt. Sobald sie aus diesem herausgewachsen sind, entfaltet sich ihre große Spreite mit verdickter Mittelrippe. Die Blätter können $8 \mathrm{~m}$ lang und $70 \mathrm{~cm}$ breit werden. Sie reißen seitlich leicht ein und bieten dann dem Wind oder heftigen Regengüssen weniger Widerstand. Bananenblätter von im Freien stehen-

Abb. 1 (Seite 148): Musa x paradisiaca mit überhängen$\mathrm{dem}$, fast bis zum Boden reichenden Blütenstand.

Abb. 2 (oben): Auf Sizilien im Freien kultivierte, blühende Obstbanane mit durch Wind zerfetzten Blättern. den Pflanzen sind deshalb meist stark zerschlitzt. Dies beeinträchtigt jedoch nicht die Funktionstüchtigkeit des Blattes, da aufgrund der parallel angeordneten Blattadern alle Teile des Blattes von der Mittelrippe ausgehend versorgt werden können. Zerschlitzte Blätter bieten Wind weniger Widerstand und sind letztendlich langlebiger.

Die Blattbildung bei den Obstbananen ist temperaturabhängig. Bei optimalen Ausgangsbedingungen wird im Ein- oder Zwei-Wochentakt jeweils ein neues Blatt hervorgebracht. In tropischen Regionen weist es eine Lebensdauer von rund 100-110 Tagen auf (Franke 1994). Die Blätter sind reich an stabilisierenden $\mathrm{Fa}$ sern, die besonders bei der Faser-Banane (Musa textilis) kommerziell gewonnen und nach Bearbeitung unter der Bezeichnung Manilahanf in den Handel gebracht werden. Die Fasern eignen sich z. B. zur Herstellung von Schiffstauen, Kabeln und Säcken. Auch Teebeutel werden aus Bananenpapier (Manilapapier) hergestellt. Die attraktiven, mitunter wie künstlich ver- 

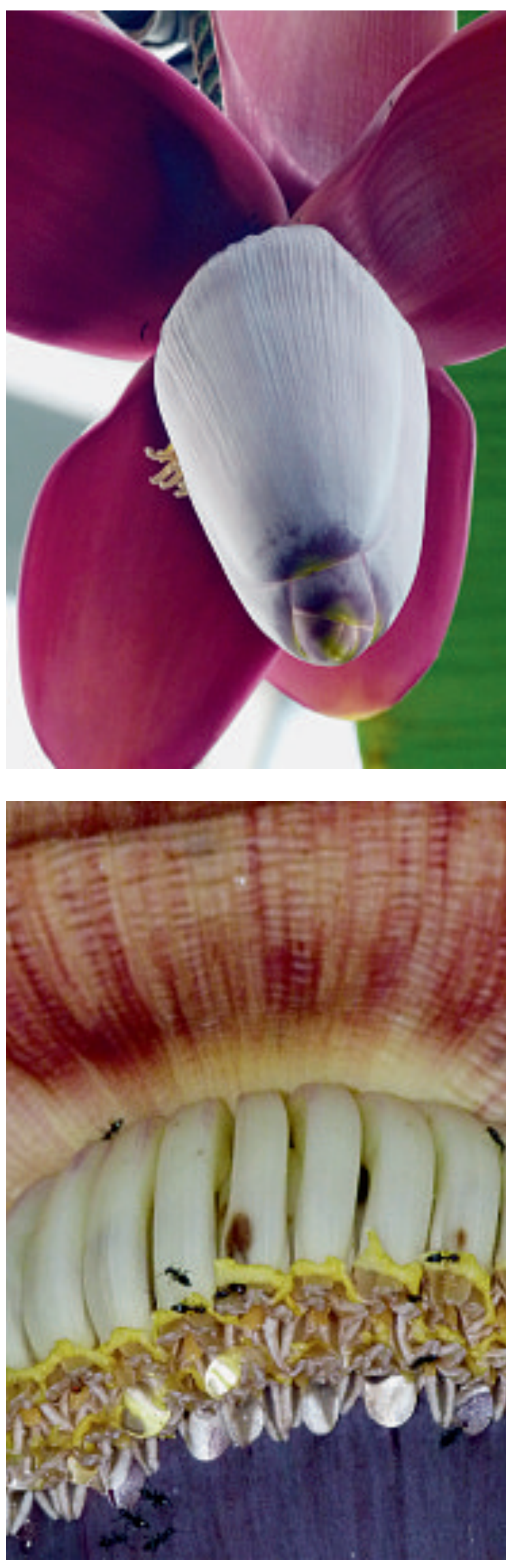

woben wirkenden Strukturen der Blattfasern von Kulturbananen werden auf Gran Canaria zur Herstellung von Schmuckketten verwendet.

\subsection{Blütenstand und Blüten}

Wenn das Rhizom nach mehreren Monaten ausreichend Nährstoffe eingelagert hat, wird der Blütenstand gebildet. Im Gegensatz zu den Blättern, die vom Vegetationspunkt als seitliche Organe abgegliedert werden, beendet der Blütenstand nun das Wachstum des Sprosses. Der Vegetationskegel wird aufgebraucht und das Wachstum der Sprossachse abgeschlossen.

Nach einigen Monaten wächst bei den Obstbananen die Sprossachse durch das Zentrum des hohlen Scheinstammes hindurch und bildet einen spektakulären Blütenstand, an dem später die Früchte reifen. Nach dem Blühen und Fruchten sterben die oberirdischen Teile der Bananenstaude ab. Spätestens dann haben sich an der Basis bereits Tochter-Pflanzen gebildet, die austreiben, wenn der fruchttragende Trieb abstirbt oder abgeschnitten wird. Die Pflanze stirbt also nach der Blüte nicht komplett $\mathrm{ab}$, wie dies manchmal zu lesen ist.

Die Blütenstände der Kulturbananen sind Ähren. Sie enthalten wenig Festigungsgewebe, sodass die Blütenstandsachse bei den meisten Arten weit bogig überhängt. Bei manchen $\mathrm{Ba}$ nanen kann die Spitze des Blütenstandes bis kurz über dem Boden hängen. Im basalen Teil des Blütenstandes befinden sich die weiblichen Blüten. Sie sind zu mehreren als so genannte Hände in der Achsel großer, spiralig gestellter, bei der Kulturbanane häufig dunkelviolett gefärbter Hochblätter vereinigt. Im terminalen Bereich des Blütenstandes werden die männlichen Blüten ausgebildet, die ebenfalls in der Achsel derber Hochblätter stehen. Zwischen weiblichen und männlichen Blüten können sich bei manchen Bananen zudem zwittrige Blüten

Abb. 3 (oben): Blütenstand von Musa textilis.

Abb. 4 (unten): Ausschnitt aus dem Blütenstand von Musa x paradisiaca im Tropicarium. Der von den einzelnen Blüten abgesonderte Nektar wird von Ameisen getrunken. 
befinden, die sich aber nicht zu Früchten entwickeln (GLADIS 2000).

An einem Blütenstand blühen die männlichen Blüten nach den weiblichen auf. Bei Wildbananen wird durch diesen Mechanismus Selbstbestäubung verhindert und die Bestäubung mit dem Pollen eines anderen Individuums gefördert. Bananenblüten umfassen sechs Blütenblätter, sechs Staubblätter und drei Fruchtblätter, die einen unterständigen Fruchtknoten bilden. Fünf der Blütenblätter sind miteinander verwachsen, ein einzelnes Blütenblatt ist kleiner und frei. Eines der Staubblätter ist in der Regel unfruchtbar oder völlig reduziert. Bei funktionell eingeschlechtigen Blüten sind Staub- bzw. Fruchtblätter mehr oder weniger vollständig verkümmert.

Die Blüten vieler Wildbananen werden durch Fledermäuse oder Vögel bestäubt. Arten wie z. B. Musa violascens, M. coccinea, M. velutina und $M$. mannii sind vogelblütig. Sie entwickeln mehr oder weniger aufrechte Blütenstände mit gelblichen bis roten oder rotvioletten Hochblättern, die die Blüten am Morgen freigeben. Die vogelbestäubten Blüten sind geruchlos und bilden einen wässrigen Nektar. Bananen-Arten, die von Fledertieren besucht werden (z. B. Musa acuminata), haben überhängende Blütenstände. Die Hochblätter sind hier oft dunkelrot oder schwarzviolett bis gelblich-grün gefärbt und öffnen sich am Abend. Fledermaus-Blüten duften stark, bilden reichlich Pollen und schleimigen oder geleeartigen Nektar. Fledermäuse werden aus der Ferne durch Geruch, beim Näherkommen durch Farbe, Form, Nektar- und Pollenreichtum angelockt. Die Tiere landen auf dem Blütenstand, führen ihre Schnauze in die Blüte ein und schieben dabei die Blütenblätter auseinander. Der im Blütengrund angesammelte Nektar fließt in die Spitze und wird mit der Zunge aufgenommen, die bei Fledermäusen oft pinselartig ausgebildet ist. Wenn die Fledermaus von männlichen Blüten einer anderen Bananenstaude Pollen mitgebracht hat, kann dieser auf die Narbe gelangen und so die Bestäubung erfolgen. Der reichliche Nektar der Bananenblüten lockt mitunter auch Insekten
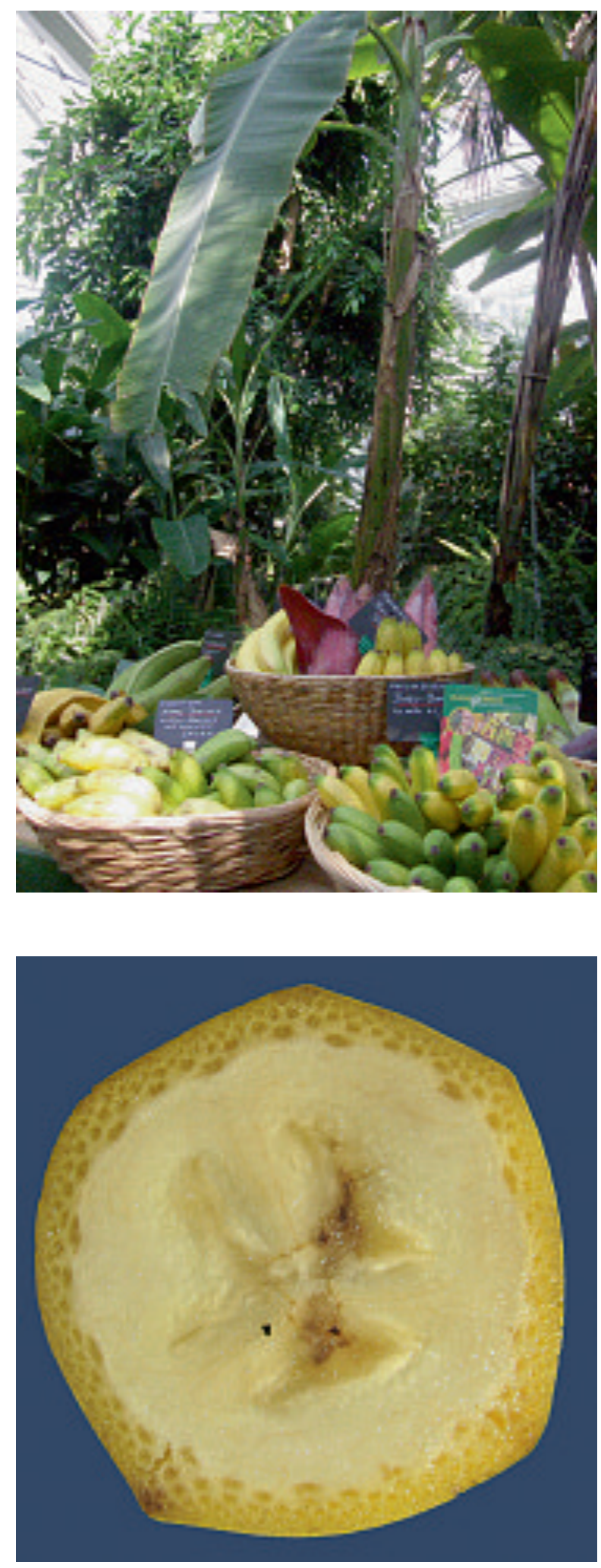

Abb. 5 (oben): Im Rahmen von Bananen-Ernteaktionen im Palmengarten werden Einblicke in die Sortenvielfalt gegeben.

Abb. 6 (unten): Querschnitt durch eine Obstbanane. Die Frucht enthält keine Samen. 


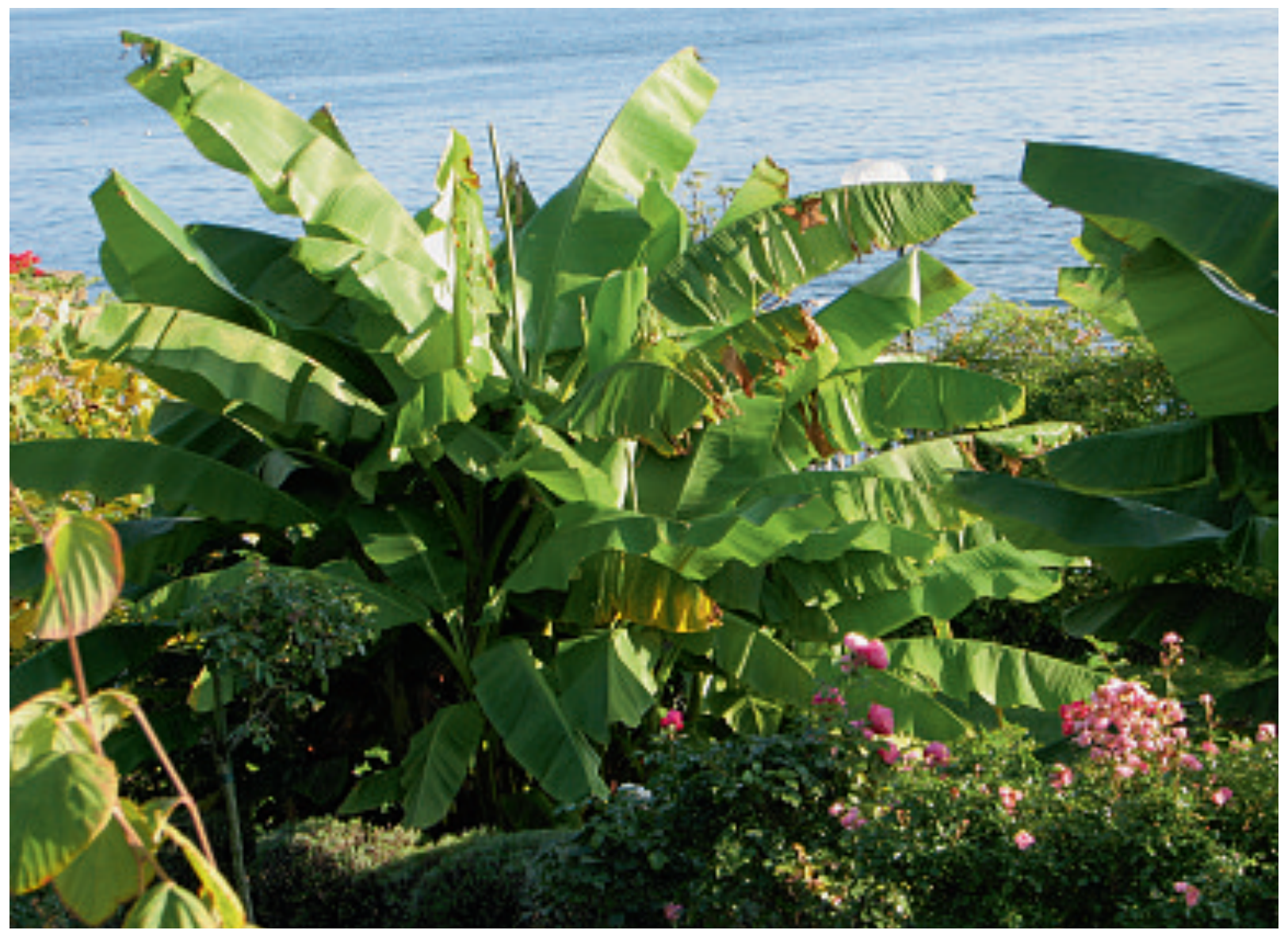

an, die allerdings wegen ihrer geringen Körpergröße nicht als Bestäuber fungieren. In Gewächshäusern kann beobachtet werden, dass Ameisen, die in Tropenhäusern allgegenwärtig sind, an den Blüten sitzen und die dicken Nektartropfen trinken.

\subsection{Früchte}

Bei den Essbananen entstehen die samenlosen Früchte ohne Bestäubung (Parthenokarpie = Jungfernfrüchtigkeit), wohingegen Wildbananen i. A. harte schwarze Samen enthalten. Mitunter gibt es aber auch unter den Wildformen samenlose Früchte, weil Parthenokarpie innerhalb der Gattung Musa nicht selten ist. Im Fruchtfleisch der Essbananen sind verkümmerte Reste der Samenanlagen als braune Pünktchen erkennbar.

Junge Bananenfrüchte sind noch gerade. Während der Reife krümmen sie sich aufwärts. Dadurch werden die darunter gelegenen jüngeren Blüten nicht mehr von den heranwachsenden Früchten bedeckt. Durch die zunehmende
Krümmung der Früchte wird Bestäubern der Zugang zu den noch unbestäubten Blüten ermöglicht.

Essbananen sind Schließfrüchte (Beerenfrüchte), die sich aus drei Fruchtblättern aufbauen. Bei einigen Bananen-Arten wie z. B. Musa velutina öffnen sich die Früchte jedoch zum Zeitpunkt der Samenreife. Die Fruchtschale löst sich in zahlreichen Längsstreifen unabhängig von den Grenzen der Fruchtblätter vom Fruchtfleisch ab, sodass das weiße Fruchtfleisch weit aus der geöffneten Frucht herausragt. Demnach trifft die Definition der Bana-

Abb. 7 (oben): Musa basjoo, ganzjährig im Freien kultiviert in Meersburg am Bodensee.

Abb. 8 (Seite 153 oben): Ausschnitt eines

Blattes von Musa basjoo.

Abb. 9 (Seite 153 Mitte): Blütenstand von Musa basjoo.

Abb. 10 (Seite 153 unten): Blütenstand von Musa basjoo im Detail. 
nen-Frucht als Schließfrucht nicht auf alle Musa-Arten zu. Das samenreiche Fruchtfleisch geöffneter Früchte wird überwiegend von größeren Vögeln (z. B. Tukane, Papageien) gefressen, die somit zur Ausbreitung der Samen beitragen. Die Früchte eines Fruchtstandes öffnen sich dabei nicht synchron, sondern nach und nach. Durch die rote Fruchtwandfarbe der übrigen, noch geschlossenen Bananen werden weitere Vögel angelockt.

Die faserige Fruchtwand (Bananenschale) ist lederig und dick, bei der Kulturbanane erreicht sie Stärken bis $6 \mathrm{~mm}$. Aufgrund der Fasern ist es möglich, die Fruchtwand abzuziehen, ohne sie dabei zu zerreißen. Neben den zahlreichen Fasern ist die Fruchtwand auch von einer Vielzahl von milchröhrenartigen Gerbstoffschläuchen durchsetzt. Wird die Fruchtwand verletzt, kann Latex austreten. Die darin enthaltenen Tannine oxidieren an der Luft und färben die Bananenschale braun bis schwarz (vgl. auch Lieberei \& Reisdorff 2007).

\subsection{Vielfalt der Bananen-Sorten}

Die Sortenvielfalt der essbaren (samenlosen!) Bananen ist riesig. Sie variieren in ihrer Größe, Form, Farbe der Fruchtschale oder dem Stärkebzw. Zuckergehalt. Obst- oder Dessertbananen, bei denen die Stärke mit zunehmender Reife zu einem großen Teil verzuckert, schmecken süßlich. Während der Reifung von Obstbananen wird die Stärke in der Frucht zu Zucker abgebaut. Sie schmecken daher mehr oder weniger süß und werden hauptsächlich roh verzehrt. Daneben werden Obstbananen auch gekocht, gebraten, zu Saft oder Mark verarbeitet sowie zur Herstellung von alkoholischen Getränken (Bier, Likör) verwendet. Neben den großfrüchtigen Sorten der Dessertbananen (z. B. 'Cavendish', das ist eine der häufigsten Sorten der normalen Essbananen) werden gelegentlich auch kleinfrüchtige Sorten verkauft, die sog. Gourmet-Bananen. Sie zeichnen sich dadurch aus, dass zur Reifezeit noch mehr Stärke in Zucker umgewandelt wird und sie daher noch süßer schmecken. Die Gourmet-Bananen werden meist in Plastikbeuteln eingeschweißt angeboten. Dadurch wird ein rasches Ausreifen
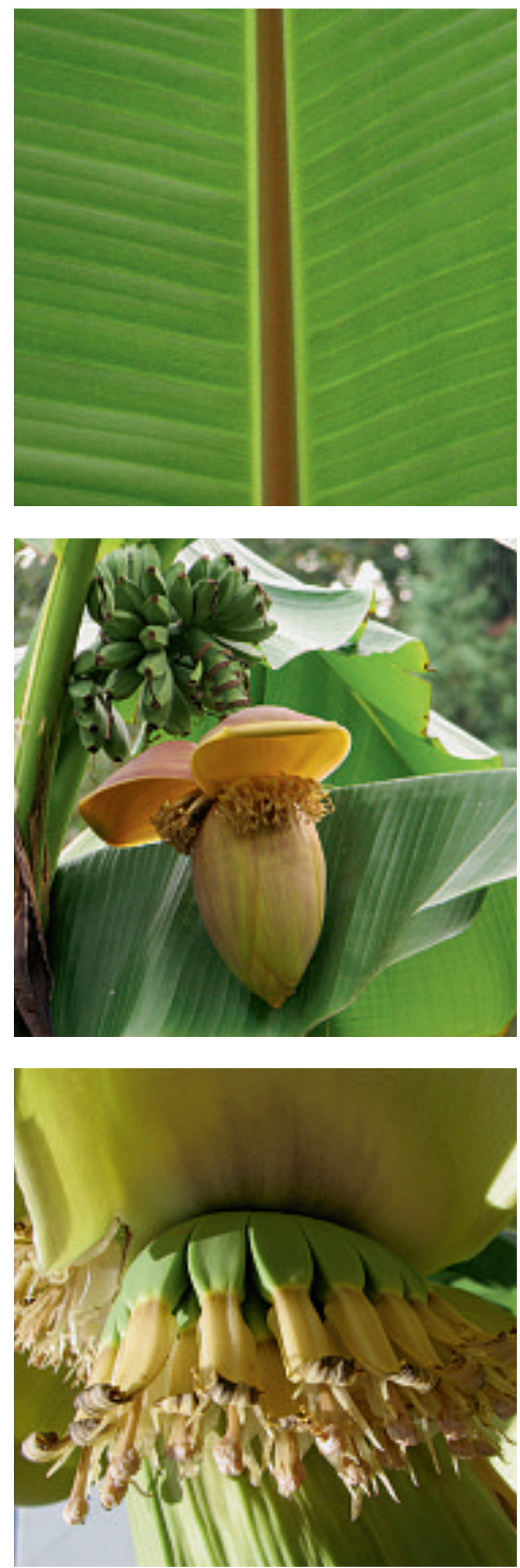

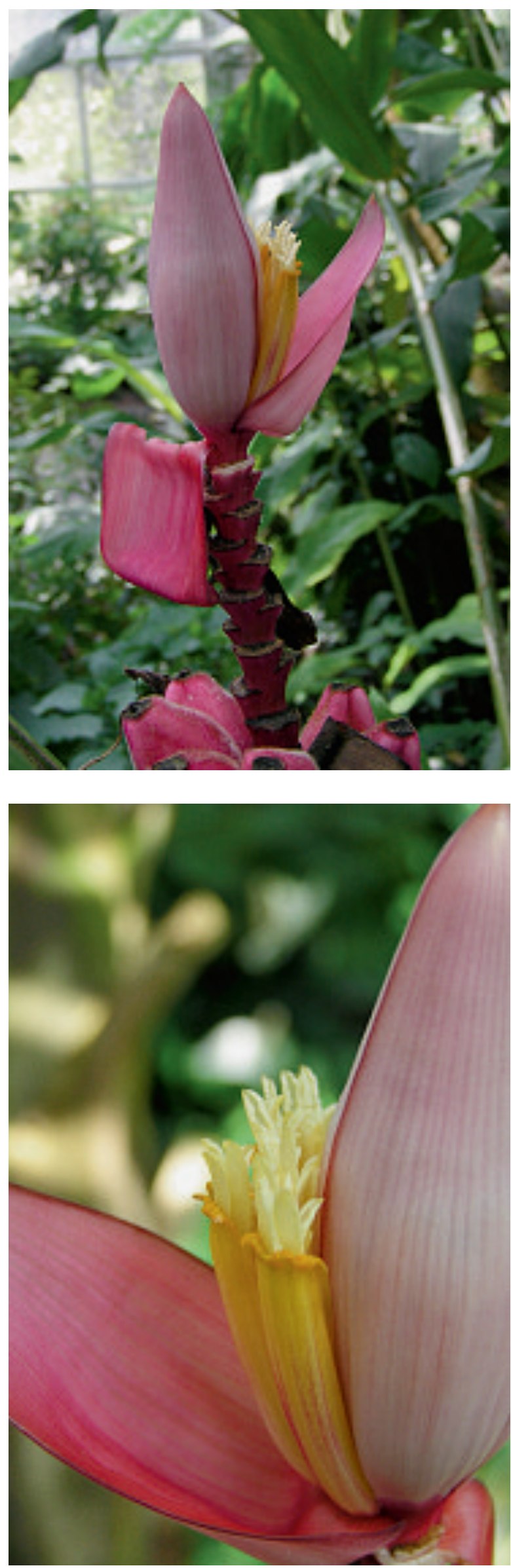

der Bananen erzielt, das durch die Abgabe von Ethylen durch die Früchte gefördert wird. Die manchmal bei uns angebotenen roten Bananen haben eine Schale, die mit zunehmender Reife dunkelrot bis rötlich violett wird. Das Fruchtfleisch ist lachsfarben und schmeckt süß (vgl. auch Nowak \& Schulz 1998).

Bei den seltener bei uns erhältlichen Kochoder Stärkebananen, von denen die meisten deutlich größer als die Obstbananen sind, wird die Stärke zur Fruchtreife hin weniger stark in Zucker umgewandelt. Die Kochbanane hat deshalb ein stärkehaltiges hartes Fruchtfleisch. Grüne Kochbananen schmecken mehlig bis fade, die reiferen gelb- bis schwarzschaligen sind etwas süßer. Kochbananen werden vor dem Verzehr gebacken, gekocht, gebraten oder getrocknet. Getrocknete Bananen werden weiter zu Bananenmehl verarbeitet, welches z. B. Getreidemehl bei der Brotherstellung beigefügt werden kann. In vielen tropischen Ländern zählen Kochbananen zu den Grundnahrungsmitteln.

Alle Kulturbananen, egal ob Obst- oder Kochbananen, stammen von den beiden Wildarten Musa acuminata (Genom A) und Musa balbisiana (Genom B) ab. Die Kulturbananen lassen sich nach der Herkunft ihres Erbgutes in Gruppen einteilen. Die wichtigsten Genom-Kombinationen gängiger Obstund Kochbananen sind AA (vor allem kleine, süße Obstbananen), AB (kleine Bananen, z. B. 'Lady's Finger'), AAA (große Sorten, darunter die wichtigsten Export-Obstbananen, z.B. 'Cavendish', 'Gros Michel'), AAB (lange Kochbananen, in West-Afrika und LateinAmerika beliebt: 'Plantain', 'Platano'), ABB (kurze Kochbananen; besonders in SüdostAsien verbreitet).

Abb. 11 (oben): Musa velutina im Tropicarium.

Abb. 12 (unten): Detail des Blütenstandes von Musa velutina.

Abb. 13 (Seite 155 oben): Wie kleine Sterne wirken die geöffneten Früchte von Musa velutina.

Abb. 14 (Seite 155 unten): Noch fast geschlossene Früchte von Musa velutina. 


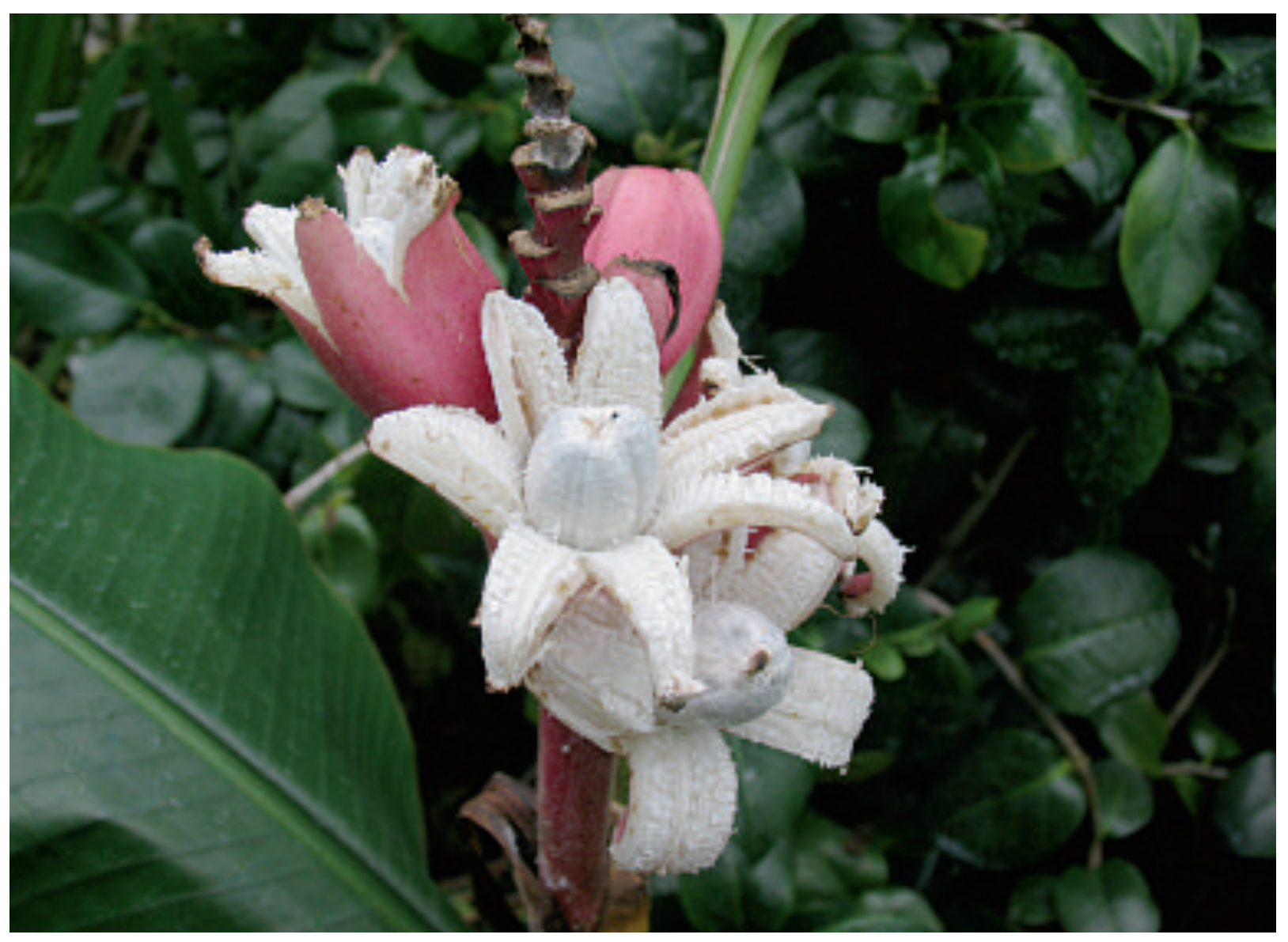

Verschiedene Sorten von Musa x paradisiaca, darunter auch 'Kongo' sowie die ähnliche Faserbanane (Musa textilis) sind im Palmengarten im Palmenhaus sowie im Tropicarium zu sehen.

\section{Bananenpflanzen für die Kultur im Freien und unter Glas}

Als Zierpflanzen gehaltene Bananenstauden werden bei uns meist als Kübelpflanzen kultiviert und im Haus überwintert. Seit einigen Jahren werden vermehrt aber auch winterharte Bananen in Gartencentern angeboten, von denen wohl die aus Süd-Japan stammende Japanische Faserbanane (Musa basjoo) die robusteste Art ist. Für eine erfolgreiche Freilandkultur benötigt Musa basjoo einen hellen und möglichst sonnigen Standort. Das Substrat sollte humos, durchlässig und vor allem gut mit Wasser und Nährstoffen versorgt sein. Wasser- und Nährstoffmangel führen zwangsläufig zu Kümmerwuchs und zu einem Entwicklungsstopp, sodass keine neuen Blätter mehr gebildet werden

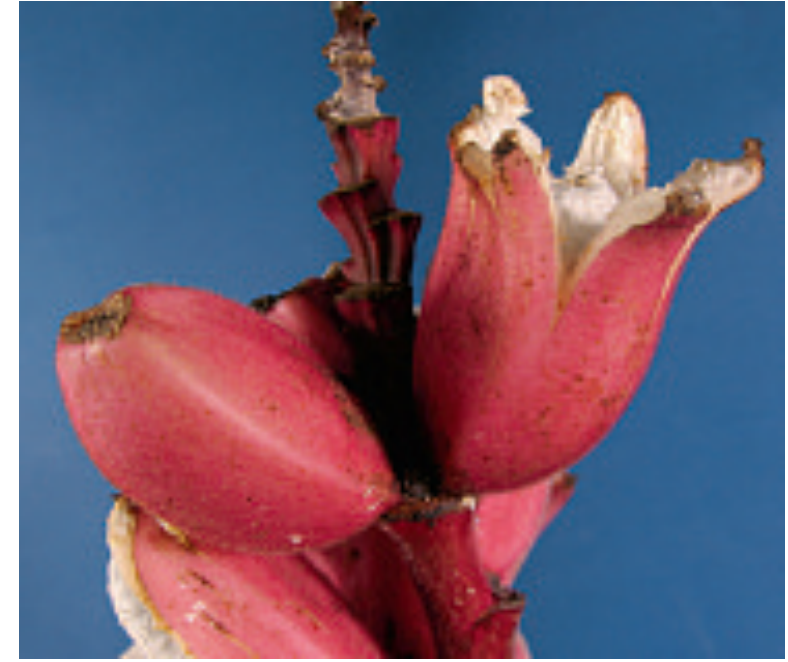

bzw. die Blätter im Scheinstamm stecken bleiben und nicht zur Entfaltung kommen.

Im Herbst werden die Bananenstauden stark zurückgeschnitten. Ein radikaler Rückschnitt wird dabei problemlos vertragen, denn der Vegetationspunkt, an dem die neuen Blätter gebildet werden, liegt kurz oberhalb der Bodenoberfläche. Einen optimalen Schutz vor 


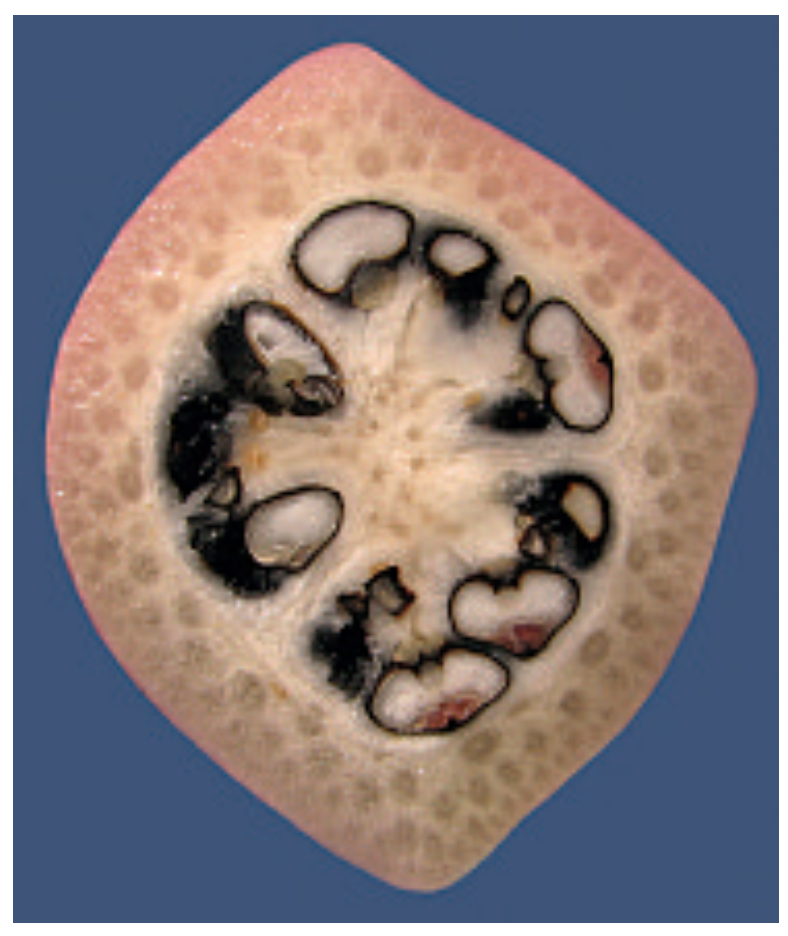

allzu tiefen winterlichen Temperaturen bietet eine großzügige Abdeckung mit Falllaub (mind. $1,5-2 \mathrm{~m}^{3}$ je Individuum). Entsprechend eingepackt können die Arten auch Extremwinter wie die letzten drei vergangenen im Freiland überstehen. So geschützt haben beispielsweise auch im Freiland des Palmengartens und im Botanischen Garten Bochum gepflanzte Individuen von Musa basjoo Temperaturminima von $-20^{\circ} \mathrm{C}$ schadlos überstanden. Im Frühjahr, wenn keine Fröste mehr zu erwarten sind, wird das Falllaub wieder entfernt. Nun sollte in das Substrat Humus eingearbeitet und die Pflanze zusätzlich mit Dünger versorgt werden. Sind ausreichend Nährstoffe vorhanden, treiben die Individuen in kürzester Zeit wieder kräftig durch und können, vorausgesetzt, das Rhizom konnte genügend Nährstoffe einlagern, dann ab Ende Juni oder Anfang Juli Blütenstände bilden.

Nicht winterfest dagegen ist Musa coccinea (Rote Zwergbanane). Sie lässt sich im Gewächshaus oder Wintergarten kultivieren und ist auch im Palmengarten im Tropicarium (Regenwälder-Haus) zu sehen. Die Staude wird nur etwa $2 \mathrm{~m}$ hoch, die Blätter erreichen Längen um $80 \mathrm{~cm}$. Ihre Blütenstände sind aufrecht und entwickeln leuchtend rote Hochblätter. Sie hal-

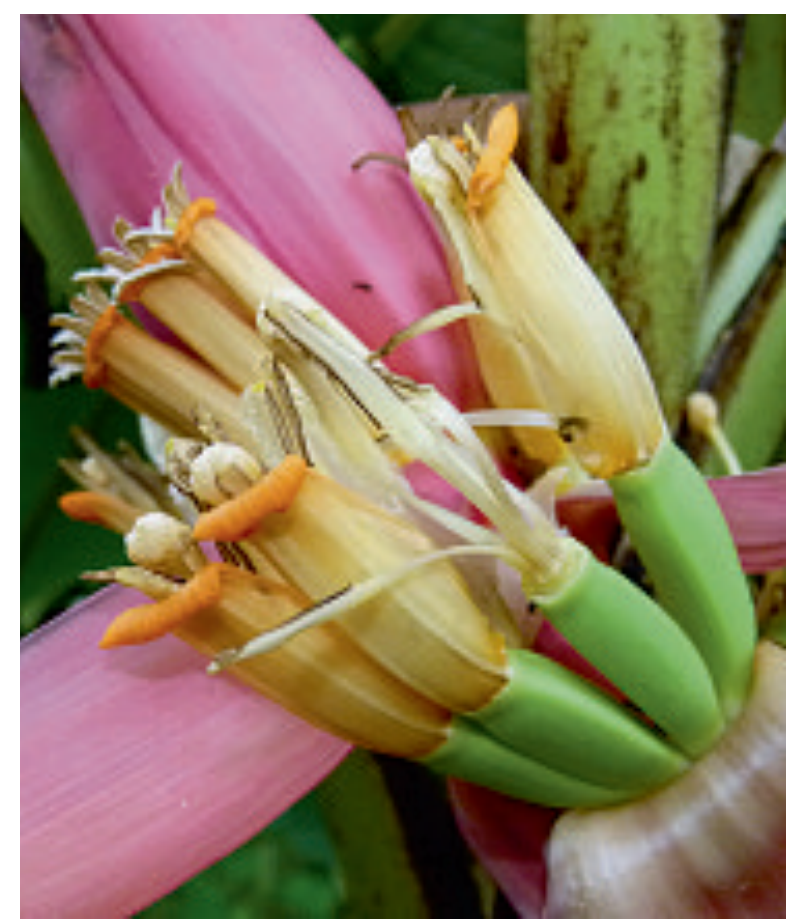

ten sich mehrere Wochen lang. Die rundlichen Früchte sind scharlachrot gefärbt.

Ebenfalls als Kübelpflanze eignet sich die Indische Zwergbanane (Musa mannii). Die mehrjährige Staude wird etwa 1,5 m hoch, hat einen dunklen Scheinstamm und gut $70 \mathrm{~cm}$ lange Blätter. Ihr Blütenstand ist zunächst aufrecht und hängt erst später etwas über. Die cremefarbenen bis gelblichen Blüten werden von rosa bis purpur gefärbten Hochblättern umgeben. Die ungenießbaren Früchte ähneln kleinen grünen Kulturbananen. Einen ähnlichen Habitus hat die Rosa Zwergbanane (Musa velutina). Von der vorherigen Art unterscheidet sie sich vor allem durch die zur Reifezeit attraktiven roten Früchte an aufrecht orientierten Fruchtständen. Wegen ihrer vielen Samen sind die Früchte nicht genießbar. Musa velutina gehört zu den wenigen Arten, die auch bei Zimmerkultur regelmä-

Abb. 15 (links): Querschnitt durch die samenhaltige Frucht von Musa velutina.

Abb. 16 (rechts): Musa mannii im Tropicarium.

Abb. 17 (Seite 157 oben): Musa coccinea im Tropicarium.

Abb. 18 (Seite 157 unten): „Bananenhain“ im Palmenhaus. 
ßig blühen und fruchten. Diese Art verträgt es gut, wenn sie im Sommer im Freien steht. Die Indische sowie Rosa Zwergbanane befinden sich im Tropicarium des Palmengartens im Monsun-Passatwälder-Haus.

\section{Literatur}

Franke, G. 1994: Nutzpflanzen der Tropen und Subtropen, Bd. 2. Spezieller Pflanzenbau. - Stuttgart. Gladis, T. 2000: Zingiberales. - In: FuKarek, F.: Urania Pflanzenreich, Blütenpflanzen 2. - Berlin.

Lieberei, R. \& Reisdorff, C. 2007: Nutzpflanzenkunde. 7. Aufl. - Stuttgart, New York.

NowaK, B. \& Schulz, B. 1998: Tropische Früchte.

Biologie, Verwendung, Anbau und Ernte. - München.
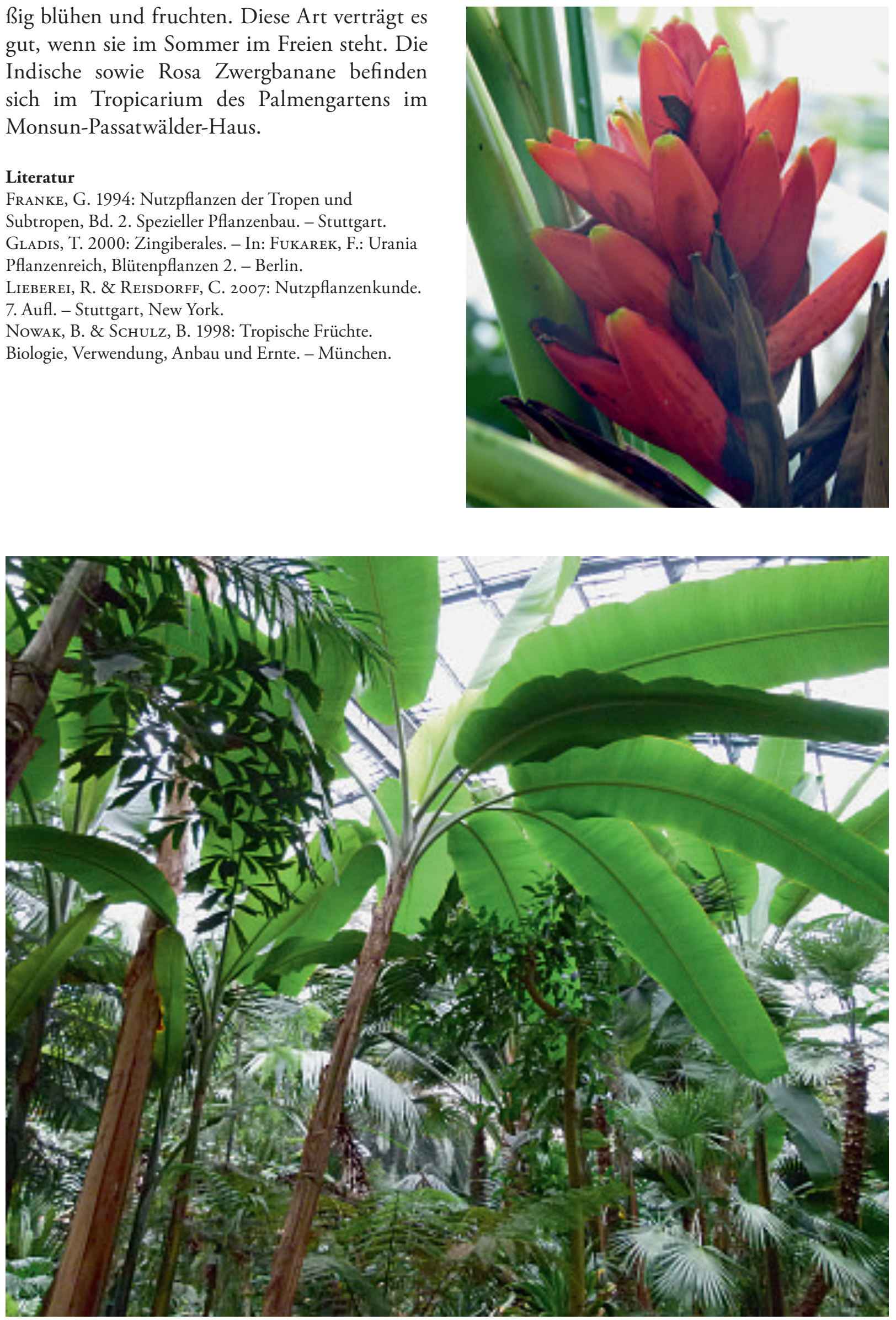